\title{
Structure démographique et régénération naturelle des peuplements naturels de Pterocarpus erinaceus Poir. (Fabaceae) des savanes de Côte d'Ivoire
}

Alice Estère GobA ${ }^{1}$

Kouamé Guillaume Kofri ${ }^{1}$

Raoul Sylvère SIE $\hat{E}^{1}$

Léonie Clémence KouonoN ${ }^{1}$

Yeboa Alexis KoFfI ${ }^{2}$

${ }^{1}$ Université Nangui Abrogoua Unité de formation et de recherche en Sciences de la Nature

Pôle de recherche Production végétale 02 BP 801, Abidjan 02

Côte d'Ivoire

${ }^{2}$ Société de développement des forêts (SODEFOR)

Direction projet et financement

01 BP 3770, Abidjan 01

Côte d'Ivoire

Auteur correspondant /

Corresponding author:

Alice Estère Goba - aegoba3@yahoo.fr

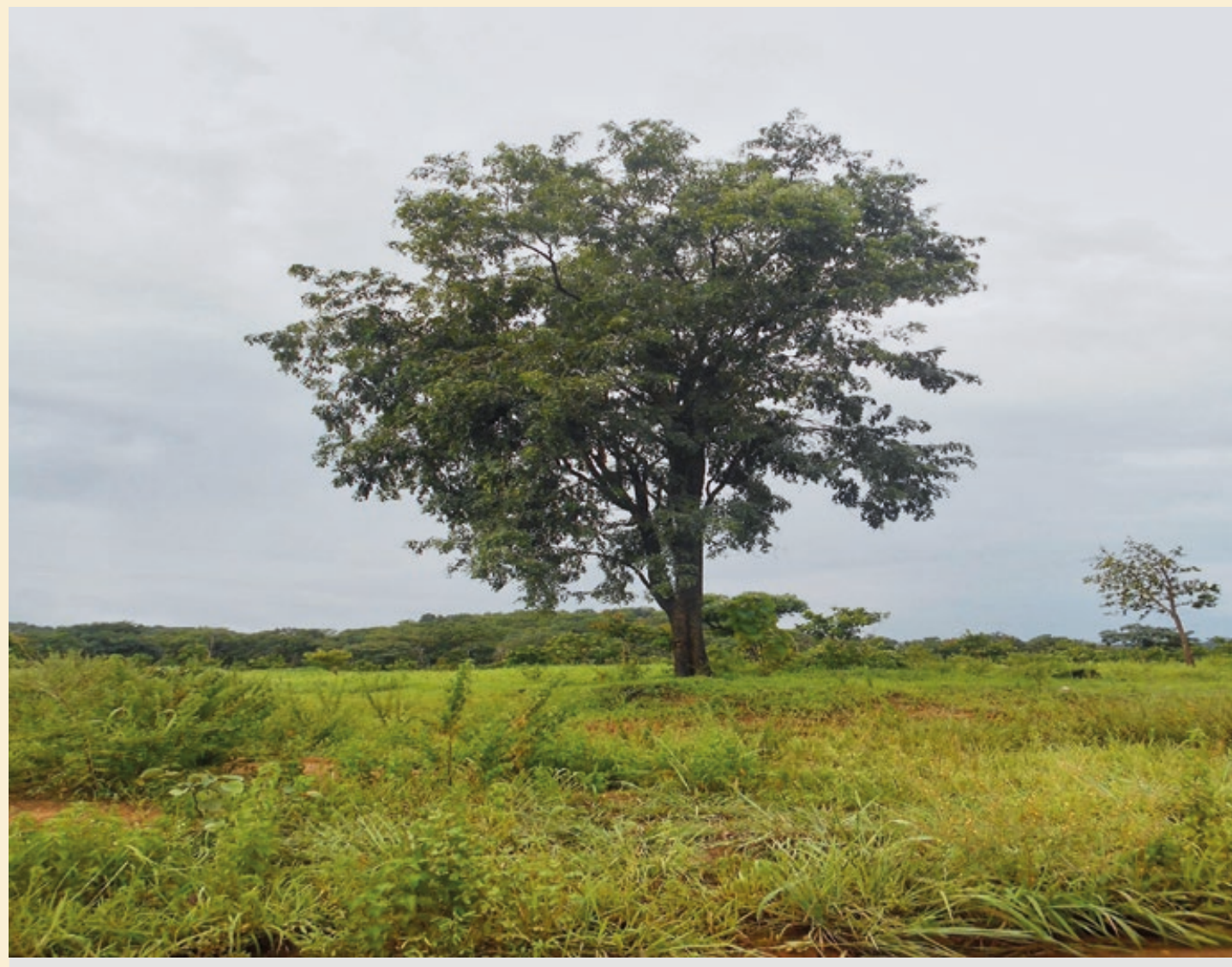

Photo 1.

Arbre de Pterocarpus erinaceus Poir. dans son habitat naturel au nord de la Côte d'Ivoire.

Photo A. E. Goba, 2017.

Doi : 10.19182/bft2019.341.a31750 - Droit d'auteur (C) 2019, Bois et Forêts des Tropiques - (c) Cirad - Date de soumission : 20 mars 2018 ;

date d'acceptation : 7 janvier 2019; date de publication : 2 juillet 2019.

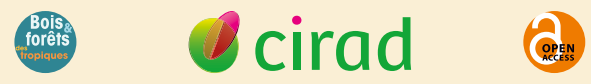

(c) $(1) \Theta$
Licence Creative Commons

Attribution - Pas de Modification 4.0 International.

Attribution-NoDerivatives 4.0 International (CC BY-ND 4.0)
Citer l'article / To cite the article

Goba A. E., Koffi K. G., Sié R. S., Kouonon L. C., Koffi Y. A., 2019. Structure démographique et régénération naturelle des peuplements naturels de Pterocarpus erinaceus Poir. (Fabaceae) des savanes de Côte d'Ivoire. Bois et Forêts des Tropiques, 341 : 5-14. Doi : https://doi.org/10.19182/ bft2019.341.a31750 


\section{RÉSUMÉ}

\section{Structure démographique et régénération naturelle des peuplements naturels de Pterocarpus erinaceus Poir. (Fabaceae) des savanes de Côte d'Ivoire}

Pterocarpus erinaceus Poir. (Fabaceae) est un arbre des forêts claires et des savanes arborées d'Afrique, très prisé pour son bois, son fourrage et ses vertus thérapeutiques, cause de sa surexploitation en Côte d'Ivoire. La présente étude analyse la structure démographique des peuplements naturels et les potentialités de régénération de cette essence en fonction de l'intensité d'exploitation dans les trois types de savanes (guinéenne, subsoudanienne et soudanienne) de la Côte d'Ivoire en vue de leur conservation. Des mesures dendrométriques (diamètre à $1,30 \mathrm{~m}$ du sol, hauteur totale et hauteur du fût de l'arbre) ont été effectuées dans 30 transects de $200 \mathrm{~m}$ de large, sur des individus de $P$. erinaceus de diamètre égal ou supérieur à $5 \mathrm{~cm}$ dans six sites. La régénération a été évaluée dans 186 placettes de $30 \mathrm{~m} \times 30 \mathrm{~m}$ équidistantes de $50 \mathrm{~m}$ à l'intérieur des transects. Les résultats révèlent que les plus grandes valeurs moyennes de densité d'arbres et de densité de régénération ont été obtenues en savane guinéenne (9,9 arbres/ ha et 33,8 tiges/ha) et subsoudanienne (12,6 arbres/ha et 25,2 tiges/ha) alors que le diamètre moyen $(33,9 \mathrm{~cm})$, la hauteur totale moyenne $(10,6 \mathrm{~m})$ et la hauteur moyenne du fût $(3,83 \mathrm{~m})$ présentent des valeurs significativement plus élevées $(p<0,001)$ en savane soudanienne. L'étude montre que l'espèce se régénère par semis naturels, rejets de souche et drageons. La distribution en classe de diamètre s'ajuste à la distribution de Weibull avec une forme en "J renversé» en savane guinéenne et subsoudanienne et en "cloche» en savane soudanienne avec des arbres de taille moyenne. Hormis l'aire protégée de Kouassi-Ndawa, les cinq autres peuplements sont soumis à diverses pressions anthropiques (coupes, écorçage et émondage).

Mots-clés : Pterocarpus erinaceus, conservation, paramètres dendrométriques, régénération, structure démographique, Côte d'Ivoire.

\section{ABSTRACT}

\section{Demographic structure and natural regeneration of natural stands of Pterocarpus erinaceus Poir. (Fabaceae) in the savannah regions of Côte d'Ivoire}

Pterocarpus erinaceus Poir. (Fabaceae) grows in Africa's sparse woodlands and tree savannahs and is a valued species for its wood, fodder and causes of its overexploitation in Côte d'Ivoire. This study analyses the demographic structure of natural stands of this species and potential for its natural regeneration according to logging intensity in the three types of savannah (Guinean, sub-Sudanian and Sudanian) in Côte d'Ivoire, with a view to their conservation. Dendrometric measurements (diameter at $1.30 \mathrm{~m}$ in height, total height and bole height) were made in 30 transects $200 \mathrm{~m}$ in width of individual $P$. erinaceus trees with a diameter of $5 \mathrm{~cm}$ or more in six sites. Regeneration was assessed in $18630 \mathrm{~m} \times 30 \mathrm{~m}$ plots $50 \mathrm{~m}$ apart within the transects. The results show that the highest average tree and regeneration densities were in Guinean (9.9 trees/ha and 33.8 stems/ ha) and sub-Sudanian savannah (12.6 trees/ha and 25.2 stems/ha) whereas the average diameter $(33.9 \mathrm{~cm})$, average total height $(10.6 \mathrm{~m})$ and average bole height $(3.83 \mathrm{~m})$ were significantly greater $(p<0.001)$ in Sudanian savannah. The study shows that the species regenerates by natural seeding, stump shoots and suckering. The distribution of diameter classes matches the reverse J-shaped Weibull curve in Guinean and sub-Sudanian savannah, and forms a bell curve in Sudanian savannah with average-sized trees. Except for the Kouassi-Ndawa protected area, the five other stands are affected by various human pressures (felling, debarking and branch cutting).

\section{Keywords: Pterocarpus erinaceus,} conservation, dendrometric parameters, regeneration, demographic structure, Côte d'Ivoire.
A. E. Goba, K. G. Koffi, R. S. SiÉ, L. C. Kouonon, Y. A. KofFI

\section{RESUMEN}

\section{Estructura demográfica y regeneración natural de las masas naturales de Pterocarpus erinaceus Poir. (Fabaceae) de las sabanas de Costa de Marfil}

Pterocarpus erinaceus Poir. (Fabaceae) es un árbol de los bosques claros y de las sabanas arboladas de África, muy apreciado por su madera, sus pastos y sus virtudes terapéuticas, de aquí su sobreexplotación en Costa de Marfil. Este estudio analiza la estructura demográfica de las masas naturales y las potencialidades de regeneración de esta especie en función de la intensidad de explotación en los tres tipos de sabana (guineana, subsudanesa y sudanesa) de la Costa de Marfil en vistas a su conservación. Se efectuaron mediciones dendrométricas (diámetro a 1,30 m del suelo, altura total y altura del fuste del árbol) en 30 transectos de $200 \mathrm{~m}$ de ancho, en individuos de $P$. erinaceus de diámetro igual o superior a $5 \mathrm{~cm}$ en seis enclaves. La regeneración se evaluó en 186 parcelas de ensayo de $30 \mathrm{~m} \times 30 \mathrm{~m}$ equidistantes $50 \mathrm{~m}$ en el interior de los transectos. Los resultados revelan que los mayores valores medios de densidad arbórea y de densidad de regeneración fueron obtenidos en la sabana guineana (9,9 árboles/ha y 33,8 tallos/ha) y subsudanesa (12,6 árboles/ha y 25,2 tallos/ha), mientras que el diámetro medio $(33,9 \mathrm{~cm})$, la altura total media $(10,6 \mathrm{~m})$ y la altura media del fuste $(3,83 \mathrm{~m})$ presentan valores significativamente más elevados $(p<0,001)$ en la sabana sudanesa. El estudio muestra que la especie se regenera mediante semillas naturales, estolones epigeos y estolones subterráneos. La distribución por clase de diámetro se ajusta a la distribución de Weibull con una forma de "I inversa» en la sabana guineana y subsudanesa, y con forma de "campana» en la sabana sudanesa con los árboles de talla media. A excepción de la zona protegida de Kouassi-Ndawa, las cinco otras poblaciones están sometidas a diversas presiones antrópicas (podas, raspado y escamonda).

Palabras clave: Pterocarpus erinaceus, conservación, parámetros dendrométricos, regeneración, estructura demográfica, Costa de Marfil. 


\section{Introduction}

La Côte d'Ivoire a perdu près de $84 \%$ de son couvert forestier entre 1960 et 2000 (Koné et al., 2014). Les activités anthropiques (agriculture extensive, orpaillage et exploitation incontrôlée du bois-énergie et du bois d'œuvre) dues à la croissance démographique galopante en sont les causes principales (MEDD, 2016). La situation s'est dégradée cette dernière décennie car la pression s'est accentuée sur les rares espèces ligneuses des savanes à fort potentiel socio-économique (BNETD, 2015), parmi lesquelles figure Pterocarpus erinaceus Poir. (Fabaceae) (photo 1). Communément appelé bois de vène, palissandre du Sénégal, teck africain ou bois rouge, $P$. erinaceus est une espèce endémique multi-usages des zones guinéo-soudaniennes et soudano-sahéliennes (Sylla et al., 2002 ; Arbonnier, 2004 ; Ouédraogo et al., 2006). En Côte d'Ivoire, son bois est utilisé en ébénisterie et pour la confection d'instruments de musique (balafons, n'goni et djembés). C'est l'une des espèces ligneuses du nord du pays les plus utilisées dans l'alimentation des bovins (Silué et al., 2014). L'écorce, les feuilles et les racines sont également utilisées en pharmacopée pour guérir plusieurs affections dont l'anémie, la toux, la dysenterie, le paludisme et la fièvre infantile (Ouédraogo et al., 2011, 2012 ; Segla et al., 2015a).

$P$. erinaceus est exploité dans les formations naturelles en Afrique de l'Ouest et en particulier en Côte d'Ivoire, en opposition aux principes d'aménagement (Ouédraogo et al., 2006 ; Adjonou et al., 2010). La Côte d'Ivoire vient en troisième position des pays ouest-africains où l'exploitation du bois de vène s'est accentuée durant cette dernière décennie (CITES, 2017). Avec la fragilisation de l'ordre public en Côte d'Ivoire en 2002, P. erinaceus a fait l'objet de contrebande, l'exportation illégale s'étant accrue en direction des pays asiatiques (ONU, 2014 ; Interpol, 2016). L'exportation de cette espèce menacée de disparition y est interdite depuis 2013 (décret n² 2013-508 du 25 juillet 2013 portant interdiction de la coupe, du transport, de la commercialisation et de l'exportation du bois de vène). Dans le contexte actuel de surexploitation du milieu naturel, des stratégies de conservation et de protection de cette espèce mériteraient d'être mises en œuvre en Côte d'Ivoire.

Cette étude conduite dans ce même pays visait à analyser les structures démographiques des peuplements naturels de $P$. erinaceus et ses potentialités de régénération en fonction de l'intensité d'exploitation dans les trois types de savane (soudanienne, subsoudanienne, guinéenne), afin de contribuer à leur gestion durable. Il s'agissait spécifiquement de décrire les caractéristiques dendrométriques des peuplements naturels de cette espèce, d'analyser leur structure et, enfin, de déterminer leurs potentialités naturelles de régénération.

\section{Méthodologie}

\section{Site d'étude}

L'étude a été réalisée dans la savane de Côte d'Ivoire (partie nord du pays), aire naturelle de distribution de $P$. erinaceus. En fonction des variations des facteurs climatiques et de la végétation se distinguent la savane soudanienne, la savane subsoudanienne et la savane guinéenne (FAO, 2002). Dans chacune de ces zones, des sites ont été choisis afin de caractériser la variabilité écologique des peuplements naturels de $P$. erinaceus en fonction du degré d'exploitation de l'espèce (figure 1).

Dans la savane soudanienne, deux sites ont été prospectés : l'aire protégée d'Ouarigué (58 000 ha) située entre $9^{\circ} 61^{\prime}$ et $9^{\circ} 65^{\prime}$ de latitude Nord et entre $4^{\circ} 89^{\prime}$ et $4^{\circ} 96^{\prime}$ de longitude Ouest et le site de Téguirdouo (village à $5 \mathrm{~km}$ de Bouna) situé entre $9^{\circ} 25^{\prime}$ et $9^{\circ} 28^{\prime}$ de latitude Nord et entre $2^{\circ} 92^{\prime}$ et $2^{\circ} 95^{\prime}$ de longitude Ouest. Ces sites se distinguent par la présence de pâturages et d'exploitations agricoles. Le climat de la zone de type soudanais est marqué par une longue saison sèche (d'octobre à juin) et une courte saison des pluies (de juillet à septembre). La zone est caractérisée par une pluviométrie annuelle comprise entre 700 et $1200 \mathrm{~mm}$ (Soro, 2011) avec la présence intermittente de l'harmattan de décembre à février. Les températures varient

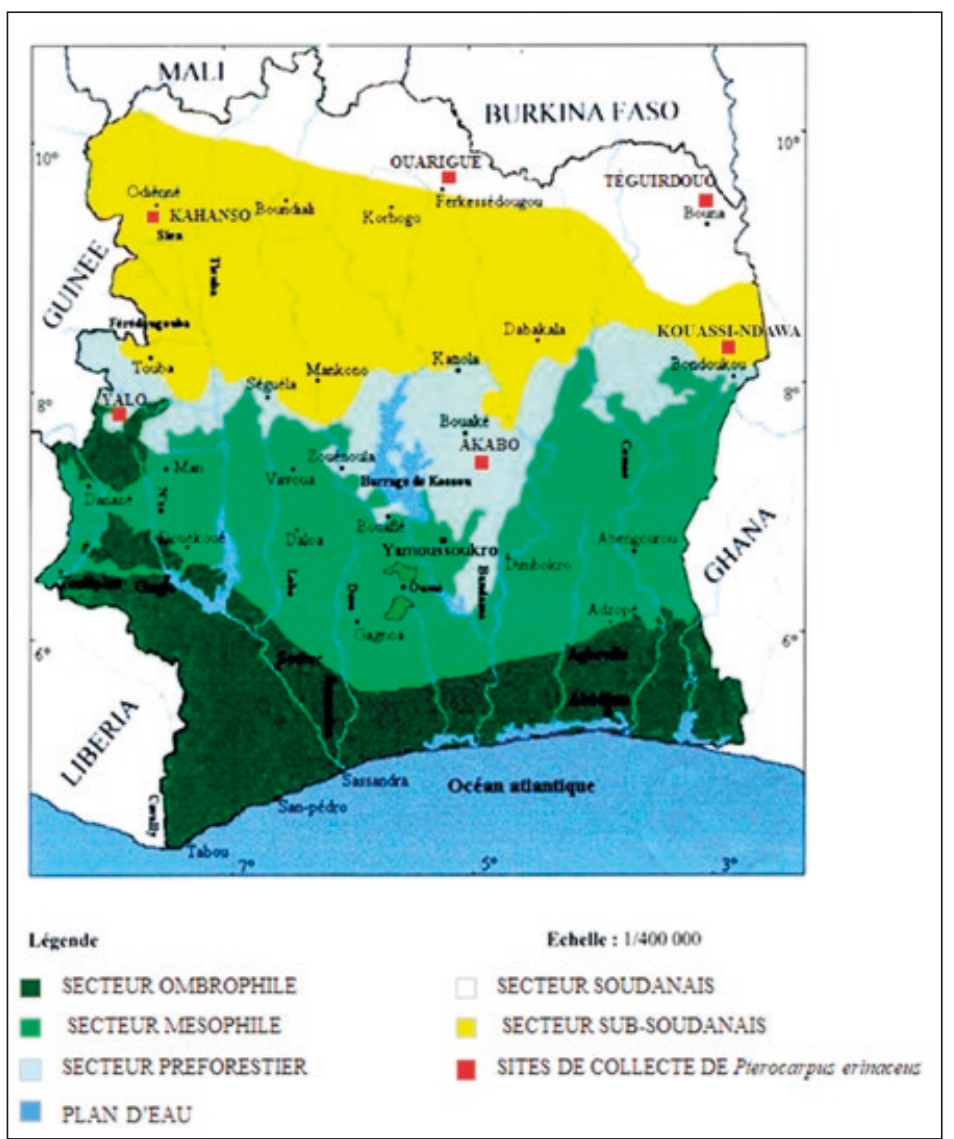

Figure 1.

Végétation de la Côte d'Ivoire et localisation des sites de collecte de données sur Pterocarpus erinaceus Poir. (Guillaumet et Adjanohoun, 1971 ; carte modifiée). 
entre 28 et $32^{\circ} \mathrm{C}$ (FAO, 2002). La végétation est constituée de savanes arbustives et herbeuses avec des galeries forestières le long des cours d'eau (FAO, 2002). Les sols sont ferrallitiques ou ferrugineux tropicaux (Roose, 1979).

Dans la savane subsoudanienne, l'aire protégée de Kahanso (7 400 ha) située sur la latitude $9^{\circ} 17^{\prime}$ 'Nord et la longitude $7^{\circ} 37^{\prime}$ ' Ouest et celle de Kouassi-Ndawa située sur la latitude $8^{\circ} 13^{\prime}$ Nord et la longitude $2^{\circ} 90^{\prime}$ Ouest (300 ha) constituent nos sites d'étude. Le site de Kahanso, peu conservé, est marqué par la présence de pâturages. En revanche, Kouassi-Ndawa est un site bien conservé. Cette zone appartient au secteur subsoudanien caractérisé par des forêts claires et des savanes arborées qui s'étendent sur des sols ferrallitiques (Roose, 1979). Le climat est de type soudano-guinéen, placé sous l'influence de l'harmattan, avec deux saisons, une pluvieuse (4 à 5 mois) et une sèche (7 à 8 mois). Les précipitations annuelles fluctuent entre 1200 et $1600 \mathrm{~mm}$ (Soro, 2011) et surviennent en majeure partie en juillet, août et septembre. La température varie entre $16^{\circ} \mathrm{C}$ et $36^{\circ} \mathrm{C}$, avec une moyenne de $27^{\circ} \mathrm{C}$ (Ouattara et al., 2016).

Dans la savane guinéenne, deux sites ont été investigués : l'aire protégée de Yalo ( $7^{\circ} 80^{\prime}$ - $8^{\circ} 10^{\prime}$ de latitude Nord et $7^{\circ} 70^{\prime}-8^{\circ} 12^{\prime}$ de longitude Ouest) de 26800 ha de superfi-

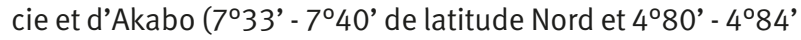
de longitude Ouest) de 2300 ha de superficie. Ces sites, positionnés respectivement à proximité des villages de Yalo et de Landonou, sont occupés par des forêts claires peu anthropisées (reboisement de teck, Tectona grandis, et présence d'exploitations agricoles). La savane guinéenne est la zone du secteur préforestier composée de forêts mésophiles (ou semi-décidues) et de savanes. Le climat est équatorial à régime bimodal, avec deux saisons des pluies espacées par deux saisons sèches (FAO, 2002). La pluviométrie moyenne annuelle est de $1200 \mathrm{~mm}$ avec une température moyenne annuelle de $27{ }^{\circ} \mathrm{C}$ (Yao et al., 2015). Les sols sont de type ferrallitique (Perraud, 1971).

\section{Collecte de données}

La méthode de collecte des données a consisté en un inventaire itinérant (échantillonnage selon des transects) (Rabiou et al., 2015a ; Ouattara et al., 2016) à l'intérieur de bandes linéaires de $200 \mathrm{~m}$ de largeur et de longueur variable. Cette méthode a été utilisée compte tenu de la faible densité apparente de $P$. erinaceus dans les formations naturelles étudiées. Elle rend compte non seulement de l'hétérogénéité structurale des peuplements mais permet aussi de recenser suffisamment d'individus afin d'obtenir une estimation fiable de la densité et de la structure démographique. Les distances parcourues ont été estimées à l'aide d'un GPS. Au total, 30 transects ont été installés dans les trois types de savane. Dans chaque transect, les mesures dendrométriques ont concerné tous les individus de $P$. erinaceus géoréférencés de diamètre égal ou supérieur à $5 \mathrm{~cm}$ à hauteur de $1,30 \mathrm{~m}$ du sol $(\mathrm{dbh}>5 \mathrm{~cm})$. En plus de la circonférence, qui a permis de déterminer le diamètre, les mesures dendrométriques ont porté sur la hauteur du fût et la hauteur totale de l'arbre. La circonférence des arbres a été mesurée avec un mètre-ruban et les hauteurs totale et du fût ont été mesurées à l'aide d'une perche graduée. Les individus dont le diamètre est inférieur à $5 \mathrm{~cm}$ ont été considérés comme faisant partie de la régénération (Mahamane et Saadou, 2008). La dynamique de l'espèce a été évaluée selon le mode de régénération dans 186 placettes de 900 m² $^{2} 30$ m x 30 m), délimitées à l'intérieur des transects, et distantes les unes des autres de $50 \mathrm{~m}$. À l'intérieur des placettes, les rejets de souche, les drageons et les semis naturels ont été dénombrés (Sokpon et al., 2006) afin d'évaluer la densité de chaque mode de régénération. L'état d'intégrité des individus a été noté afin d'évaluer l'intensité de l'exploitation de l'espèce sous forme d'écorçage, d'émondage et de coupe.

\section{Analyse des données}

Pour analyser les caractéristiques écologiques des peuplements, la densité $(\mathrm{N})$, la densité de régénération $(\mathrm{Nr})$ et la surface terrière $(\mathrm{G})$ ont été calculées. La densité $\mathrm{N}$ (arbres/ ha) est le nombre d'arbres par unité de surface. La densité moyenne de régénération $\mathrm{Nr}$ (tiges/ha) est le nombre total d'arbres de diamètre inférieur à $5 \mathrm{~cm}$ par unité de surface (placettes). La surface terrière $\mathrm{G}\left(\mathrm{m}^{2} / \mathrm{ha}\right)$ est la somme des sections transversales à hauteur de $1,30 \mathrm{~m}$ du sol, de tous les arbres du transect.

Les données calculées ont été analysées à l'aide du logiciel XLSTAT Ecology version 2018.5, en fonction des trois types de savane. L'analyse multiple de variance (MANOVA) a été réalisée pour la comparaison de l'ensemble des paramètres dendrométriques. Les analyses de variance à un facteur (ANOVA 1) ont servi à comparer les moyennes des différents paramètres dendrométriques de $P$. erinaceus dans les différents types de savane. Les tests d'ANOVA significatifs ont été suivis du test Fisher de comparaison multiple par paires (LSD) au seuil de significativité de $5 \%$. La densité des modes de régénération naturelle (rejets de souche, drageons, semis naturels) a été estimée et l'importance relative de chaque mode de régénération a été calculée en pourcentage pour les trois types de savane considérés. Une analyse de variance a été ensuite effectuée afin de comparer cette densité dans les peuplements naturels de $P$. erinaceus étudiés.

La structure démographique de l'espèce a été analysée selon 11 classes de diamètre des arbres, définies à partir d'un seuil de $5 \mathrm{~cm}$ et une amplitude de $5 \mathrm{~cm}$. Les dix classes de hauteur ont été définies à partir d'un seuil de $2 \mathrm{~m}$, avec une amplitude d'également $2 \mathrm{~m}$. Ces classes de diamètre et de hauteur ont servi à construire des histogrammes de distribution. Les structures observées ont été ajustées à la distribution théorique de Weibull à trois paramètres (Rondeux, 1999) à l'aide du logiciel XLSTAT Ecology version 2018.5. La fonction correspondante de densité de probabilité $f(x)$ se présente sous la forme suivante :

$f(x)=\frac{c}{b}\left(\frac{x-a}{b}\right)^{c-1} \exp \left[-\left(\frac{x-a}{b}\right)^{c}\right]$

où $x$ est le diamètre des arbres, a est le paramètre de position, b est le paramètre d'échelle ou de taille, et c est le paramètre de forme lié à la structure observée. 
Tableau I.

Différentes formes de la distribution de Weibull en fonction de la valeur du paramètre de forme « $\mathrm{c} »$.

\begin{tabular}{|c|c|}
\hline $\begin{array}{l}\text { Valeur du } \\
\text { paramètre « } \mathrm{c} »\end{array}$ & Interprétation \\
\hline$c<1$ & Répartition en «J renversé », caractéristique de peuplements multispécifiques et inéquiens \\
\hline$c=1$ & Diminution de la distribution exponentielle, caractéristique de peuplements en danger \\
\hline $1<c<3,6$ & $\begin{array}{l}\text { Distribution asymétrique positive, caractéristique de peuplements monospécifiques avec principalement } \\
\text { des individus de petit diamètre (jeunes espèces) }\end{array}$ \\
\hline$c=3,6$ & $\begin{array}{l}\text { Distribution symétrique (structure normale), caractéristique de peuplements monospécifiques avec des individus } \\
\text { à diamètre inégal }\end{array}$ \\
\hline c>3,6 & Distribution asymétrique négative, caractéristique de peuplements monospécifiques de vieilles espèces \\
\hline
\end{tabular}

La distribution peut prendre plusieurs formes selon la valeur du paramètre de forme (c) (Husch et al., 2003) (tableau I). Une analyse log-linéaire a été réalisée pour comparer, dans chaque cas, la distribution en diamètre (ou en hauteur) observée à la distribution théorique de Weibull avec estimation des paramètres. L'hypothèse de l'ajustement entre les deux distributions (théorique et observée) a été acceptée si la valeur de la probabilité du test était inférieure à 0,05.

\section{Résultats}

\section{Intensité de l'exploitation}

Hormis l'aire protégée de Kouassi-Ndawa en savane subsoudanienne, tous les autres peuplements sont soumis à des pressions anthropiques telles que les coupes (photo 2), l'écorçage et l'émondage (tableau II). Les plus grandes proportions d'individus coupés et écorcés sont observées en savane guinéenne. Ainsi, les sites d'Akabo et Yalo se caractérisent respectivement par 38,6\% et 35,3\% d'individus coupés et par $42,3 \%$ et $51,3 \%$ d’individus écorcés. En savane subsoudanienne (Kahanso) et soudanienne, les individus coupés et écorcés sont faiblement représentés. Cependant, $P$. erinaceus est fréquemment émondé dans ces deux types de savane pour alimenter le bétail pendant les saisons sèches où le fourrage se fait rare. L'intensité d'émondage est plus critique sur les sites d'Ouarigué $(46,7 \%)$ et de Téguirdouo (43,2 \%) en savane soudanienne, suivis du site de Kahanso (28,9\%) en savane subsoudanienne. Les faibles pourcentages d'individus émondés sont observés dans les aires protégées de Yalo et Akabo en savane guinéenne.

\section{Caractéristiques dendrométriques de Pterocarpus erinaceus}

L'analyse multiple de variance (MANOVA) réalisée pour l'ensemble des paramètres dendrométriques a montré une différence hautement significative $(p<0,001)$ entre les peuplements de $P$. erinaceus des trois types de savane. Les résultats de l'analyse de variance obtenus pour les cinq paramètres dendrométriques sont reportés dans le tableau III. Les peuplements de $P$. erinaceus de la savane guinéenne présentent une densité moyenne de 9,9 \pm 4,6 arbres/ha, statistiquement non différente $(p=0,19)$ de celle de la savane subsoudanienne $(12,6 \pm 6,3$ arbres/ha). En revanche, ces densités sont significativement plus élevées $(p<0,001)$ que celle des peuplements de la savane soudanienne $(2,37 \pm 0,77$ arbres/ha).

Le diamètre moyen des arbres de $P$. erinaceus est estimé à $23,1 \pm 10,2 \mathrm{~cm}$ en savane guinéenne, à $23 \pm 12,7 \mathrm{~cm}$ en savane subsoudanienne, et à $33,9 \pm 12,3 \mathrm{~cm}$ en savane soudanienne. Les tests statistiques indiquent que les valeurs moyennes de diamètre sont identiques $(p=0,96)$ pour les peuplements de la savane guinéenne et subsoudanienne.

Ces valeurs sont significativement différentes $(p<0,001)$ de celles de la savane soudanienne.

La hauteur moyenne des arbres des peuplements de $P$. erinaceus décroît le long du gradient climatique, les valeurs les plus élevées apparaissant en savane soudanienne $(10,6 \pm 2,3 \mathrm{~m})$. Les peuplements des savanes

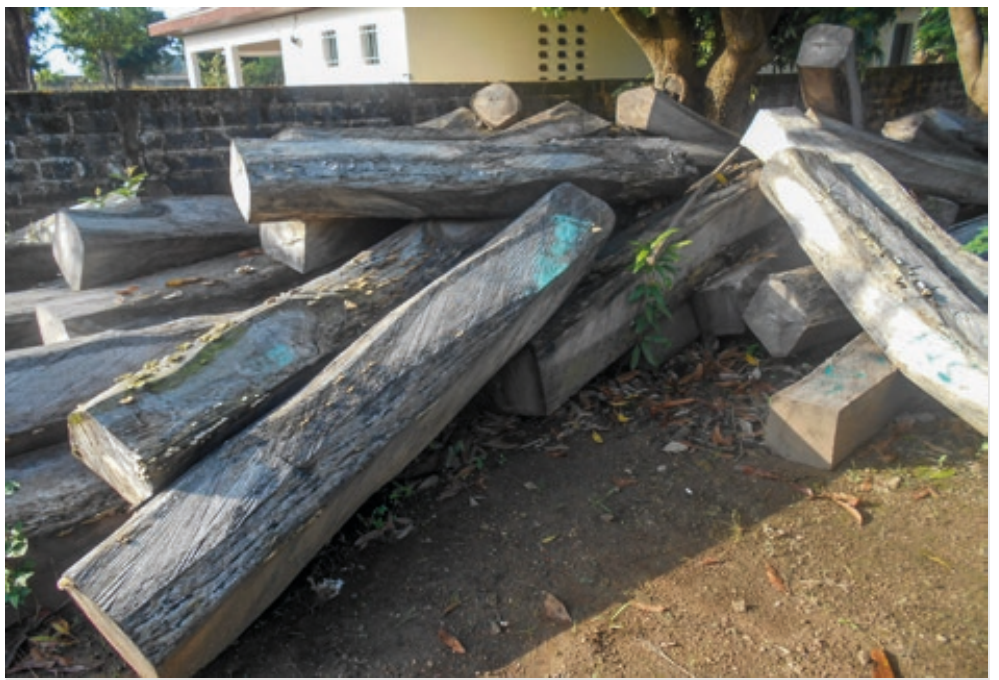

Photo 2.

Bûches de Pterocarpus erinaceus Poir. saisies par les agents des eaux et forêts et stockées dans un camp de base à Odienné (Côte d'Ivoire).

Photo A. E. Goba, 2016. 
Tableau II.

Intensité d'exploitation de Pterocarpus erinaceus dans les trois types de savane prospectés.

\begin{tabular}{l|l|c|c|c|}
\multirow{2}{*}{ Types de savane } & Sites & Coupes (\%) & Émondage (\%) & Écorçage (\%) \\
& & & & \\
\multirow{2}{*}{ Savane guinéenne } & Yalo & 35,3 & 2,91 & 51,3 \\
\cline { 2 - 5 } & Akabo & 38,6 & 3,42 & 42,3 \\
\hline \multirow{2}{*}{ Savane subsoudanienne } & Kahanso & 9,75 & 28,9 & 8,96 \\
\cline { 2 - 5 } & Kouassi-Ndawa & 0 & 0 & 7,98 \\
\hline \multirow{2}{*}{ Savane soudanienne } & Téguirdouo & 13,7 & 43,2 & 5,16 \\
\cline { 2 - 5 } & Ouarigué & 9,17 & 46,7 & \\
\hline
\end{tabular}

\section{Tableau III.}

Comparaison des valeurs moyennes \pm écart-type des paramètres dendrométriques évalués chez Pterocarpus erinaceus dans les trois types de savane prospectés.

\begin{tabular}{|c|c|c|c|c|c|c|}
\hline Types de savane & $\begin{array}{c}\text { Nombre } \\
\text { d'individus }\end{array}$ & $\begin{array}{c}\text { Densité } \\
\text { (arbres/ha) }\end{array}$ & $\begin{array}{l}\text { Diamètre } \\
(\mathrm{cm})\end{array}$ & $\begin{array}{l}\text { Hauteur } \\
\text { totale }(\mathrm{m})\end{array}$ & $\begin{array}{l}\text { Hauteur } \\
\text { du fût }(\mathrm{m})\end{array}$ & $\begin{array}{c}\text { Surface } \\
\text { terrière }\left(\mathrm{m}^{2} / \mathrm{ha}\right)\end{array}$ \\
\hline Savane guinéenne & 351 & $9,95 \pm 4,56^{\mathrm{a}}$ & $23,1 \pm 10,2^{b}$ & $9,44 \pm 2,67^{c}$ & $3,36 \pm 1,51^{b}$ & $0,41 \pm 0,24^{b}$ \\
\hline Savane subsoudanienne & 547 & $12,6 \pm 6,31^{\mathrm{a}}$ & $23 \pm 12,7^{b}$ & $10,1 \pm 3,38^{b}$ & $4,04 \pm 2,82^{\mathrm{a}}$ & $0,70 \pm 0,43^{a}$ \\
\hline Savane soudanienne & 210 & $2,37 \pm 0,77^{b}$ & $33,9 \pm 12,3^{a}$ & $10,6 \pm 2,26^{a}$ & $3,83 \pm 1,78^{a}$ & $0,29 \pm 0,14^{b}$ \\
\hline Globale & 1108 & $8,76 \pm 6,30$ & $25,1 \pm 12,6$ & $9,97 \pm 3$ & $3,78 \pm 2,31$ & $0,47 \pm 0,36$ \\
\hline Tests statistiques & - & $P<0,000$ & $P<0,0001$ & $P<0,0001$ & $P<0,0001$ & $P=0,015$ \\
\hline
\end{tabular}

subsoudanienne et guinéenne sont respectivement caractérisés par une hauteur moyenne de $10,1 \pm 3,38 \mathrm{~m}$ et de $9,44 \pm 2,67 \mathrm{~m}$. Cette différence de valeurs de hauteur est significative entre les trois types de savane $(p<0,001)$.

La hauteur moyenne du fût des arbres présente des valeurs plus grandes en savane subsoudanienne $(4,03 \pm 2,8 \mathrm{~m})$ et soudanienne $(3,83 \pm 1,8 \mathrm{~m})$. Ces valeurs statistiquement identiques $(p=0,27)$ sont en revanche supérieures à celle de la savane guinéenne (3,36 \1,5 m).

La plus grande surface terrière s'observe au sein des peuplements de la savane subsoudanienne $\left(0,70 \pm 0,43 \mathrm{~m}^{2} /\right.$ ha) et les plus faibles, statistiquement identiques $(p=0,39)$, sont relevées dans les savanes guinéenne et soudanienne (respectivement 0,41 $\pm 0,24 \mathrm{~m}^{2} /$ ha et $0,29 \pm 0,14 \mathrm{~m}^{2} / \mathrm{ha}$ ).

\section{Structure démographique des peuplements naturels de $P$. erinaceus}

La distribution des arbres de $P$. erinaceus en classes de diamètre (figure 2) observée en savane guinéenne et subsoudanienne montre une structure en «J renversé ». En savane guinéenne, cette structure est caractérisée par une prédominance d'arbres de classe de diamètre compris entre
10 et $30 \mathrm{~cm}(77,3 \%)$. Les peuplements de $P$. erinaceus de la savane subsoudanienne sont dominés par les arbres de diamètre variant de 5 à $30 \mathrm{~cm}(88,7 \%)$. La savane soudanienne est la seule zone agroécologique qui présente une structure en classe de diamètre en forme de « cloche » avec une meilleure représentation des classes de diamètre intermédiaire $(73,8$ \%) par rapport aux classes extrêmes. Toutes les structures en classe de diamètre observées s'ajustent à la distribution théorique de Weibull $(\mathrm{p}<0,05)$ avec un paramètre de forme (c) de valeur comprise entre 1 et 3,6 ; elles sont caractéristiques de peuplements monospécifiques avec principalement de jeunes individus.

Pour l'ensemble des peuplements de $P$. erinaceus des types de savane, la structure en hauteur présente une forme en cloche avec prédominance d'arbres de hauteur moyenne. En savane guinéenne et subsoudanienne, les arbres de classe de hauteur comprise entre 6 et $16 \mathrm{~m}$ sont les plus répandus, avec des proportions respectives de $94,4 \%$ et $92,8 \%$. L'analyse log-linéaire montre que ces deux distributions observées s'ajustent aux distributions théoriques de Weibull ( $p<0,05)$ avec un paramètre de forme (c) de valeur comprise entre 1 et 3,6, traduisant une prédominance de jeunes individus. Par contre, en savane soudanienne, les 
arbres de hauteur comprise entre 10 et $16 \mathrm{~m}$ sont prédominants $(83,8 \%)$ avec un paramètre de forme de la distribution théorique de Weibull supérieur à 3,6.

\section{Potentialités de régénération naturelle}

La densité globale de régénération de $P$. erinaceus varie significativement $(p<0,001)$ de 33,8 \pm 4,2 tiges/ha à $7,8 \pm 1,1$ tiges/ha avec la plus grande valeur en savane guinéenne (tableau IV). Le dénombrement des différentes stratégies de multiplication adoptées par $P$. erinaceus dans les six peuplements naturels des trois différentes savanes prospectées révèle qu'il existe trois modes de régénération chez cette espèce en Côte d'Ivoire : les semis naturels, les rejets de souche et les drageons. Les semis naturels représentent les stratégies de régénération les plus observées avec des valeurs statistiquement différentes d'un type de savane à l'autre ( $p<0,001)$. Les densités respectives des semis naturels en savane guinéenne, subsoudanienne et soudanienne sont de $24,9 \pm 6,7,19,5 \pm 6,7$ et 5,8 $\pm 1,1$ tiges/ha. Les rejets de souche, qui constituent la deuxième voie de régénération de l'espèce, présentent des valeurs statistiquement non différentes pour les trois types de savane. Les drageons ont été observés uniquement en savane guinéenne et subsoudanienne.

\section{Discussion}

\section{Pressions anthropiques sur $P$. erinaceus}

$P$. erinaceus est victime de pressions anthropiques (coupes, écorçage et émondage) dans tous les sites prospectés hormis à Kouassi-Ndawa en savane subsoudanienne. En Côte d'Ivoire, le vène est exploité pour son bois (coupe), ses vertus thérapeutiques (écorçage) et son fourrage (émondage). En savane guinéenne, les proportions d'arbres coupés et écorcés sont plus élevées (35\% à $51 \%$ ). L'espèce fournit un excellent bois d'œuvre utilisé en ébénisterie et en construction (Adjonou et al., 2010). Il sert de bois-énergie (charbon et bois de chauffe) et de bois de service (confection de mortiers, pilons, manches de daba). P. erinaceus, appelé arbre miracle par les populations locales du centre de la Côte d'Ivoire, possède également de nombreuses vertus thérapeutiques (Ouédraogo et al., 2011, 2012). Cela entraîne l'écorçage fréquent des arbres à proximité des villages. La décoction de l'écorce du vène est utilisée contre l'anémie, les ulcères, la dysenterie, le paludisme et le rhumatisme. La sève de l'écorce permet de lutter efficacement contre les dermatoses. Les feuilles sont administrées en décoction pour traiter la fièvre infantile et la bronchite, et en infusion dans des rituels visant à chasser les mauvais esprits.

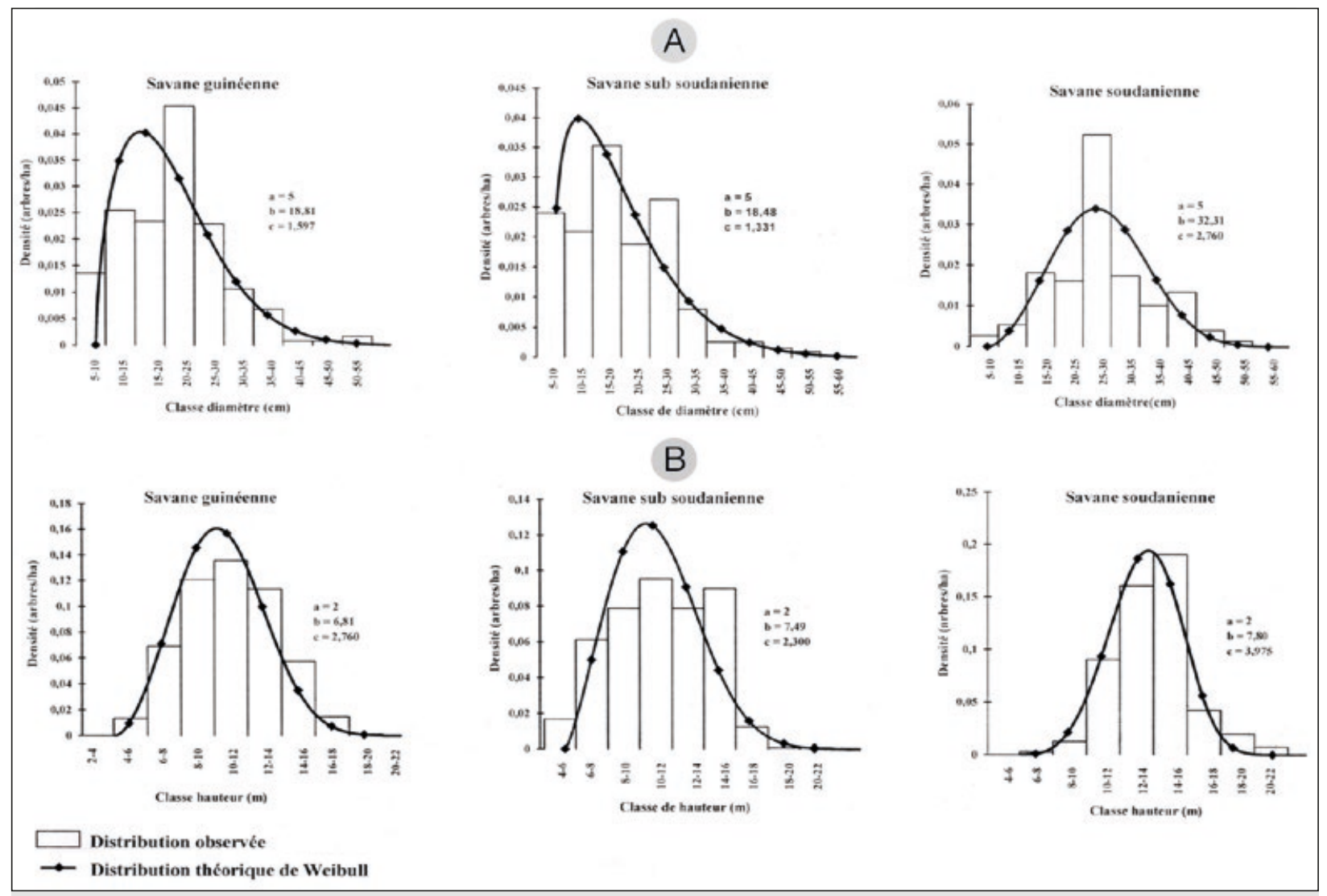

Figure 2.

Structure en diamètre (A) et en hauteur (B) de Pterocarpus erinaceus Poir. dans les trois types de savane. 
Tableau IV.

Densité et pourcentage des différents modes de régénération de Pterocarpus erinaceus dans les trois types de savane prospectés.

\begin{tabular}{|c|c|c|c|c|}
\hline \multirow{2}{*}{$\begin{array}{l}\text { Modes de } \\
\text { régénération }\end{array}$} & \multicolumn{4}{|c|}{ Densité de régénération (plantules/ha et \%) } \\
\hline & $\begin{array}{c}\text { Savane } \\
\text { guinéenne }\end{array}$ & $\begin{array}{c}\text { Savane } \\
\text { subsoudanienne }\end{array}$ & $\begin{array}{c}\text { Savane } \\
\text { soudanienne }\end{array}$ & $\begin{array}{c}\text { Tests } \\
\text { statistiques }\end{array}$ \\
\hline Moyenne globale & $33,8 \pm 4,16^{\mathrm{a}}(100 \%)$ & $25,2 \pm 6,30^{b}(100 \%)$ & $7,78 \pm 1,14^{\mathrm{c}}(100 \%)$ & $P<0,0001$ \\
\hline Semis naturels & $24,9 \pm 6,72^{\mathrm{a}}(73,7 \%)$ & $19,5 \pm 6,73^{b}(77,4 \%)$ & $5,83 \pm 1,06^{c}(74,9 \%)$ & $P<0,0000$ \\
\hline Rejets de souche & $5,07 \pm 3,59(15 \%)$ & $3,04 \pm 3,38(12,1 \%)$ & $1,95 \pm 0,41(25,1 \%)$ & $P=0,07^{n s}$ \\
\hline Drageons & $3,48 \pm 3,13^{\mathrm{a}}(10,3 \%)$ & $2,14 \pm 2,69^{b}(8,5 \%)$ & $0(0 \%)$ & $P=0,015$ \\
\hline
\end{tabular}

Sur les lignes, les valeurs moyennes suivies de la même lettre ne sont pas significativement différentes. Dans la colonne Tests statistiques, P est la probabilité associée à la comparaison des moyennes du paramètre dans les trois types de savane. Lorsque $\mathrm{P}$ est inférieur à 0,05 , la différence entre les moyennes du paramètre est significative.

En savane soudanienne et subsoudanienne, le taux d'individus émondés est nettement supérieur à celui de la savane guinéenne ( $p<0,05)$. Ce taux varie de $28,9 \%$ (Kahanso) à 43,2 \% et 46,7\% (respectivement à Ouarigué et Téguirdouo). Sur ces sites, l'espèce subit une forte pression pastorale. Les feuilles constituent un excellent fourrage pour le bétail (Silué et al., 2014). Riches en protéines et en phosphore, elles jouent un rôle essentiel dans la digestion de la cellulose de l'herbe sèche (Rabiou et al., 2015b). Ces différentes pressions anthropiques exercées sur ces peuplements impactent négativement la structure démographique et la régénération de l'espèce dans les sites prospectés.

\section{Variabilité des paramètres dendrométriques de $P$. erinaceus}

L'analyse des caractéristiques dendrométriques de $P$. erinaceus en fonction des trois types de savane en Côte d'Ivoire montre que la densité des arbres et la densité de régénération augmentent avec l'humidité du climat. Ces deux paramètres présentent des valeurs plus grandes en savane guinéenne et subsoudanienne. Cette différence pourrait être imputable à la disponibilité des ressources et aux conditions édaphiques et environnementales de chaque savane, mais aussi aux pressions anthropiques associées (Rabiou et al., 2015b). La densité globale (8,76 arbres/ha) est, en revanche, inférieure à celles obtenues par Kakaï et al. (2008) au Bénin (22,6 arbres/ha) et par Segla et al. (2015b) au Togo (57 à 76,5 arbres/ha) sous les mêmes latitudes. Cela s'explique manifestement par la différence des pressions anthropiques et des conditions écologiques (CITES, 2015).

Les gros diamètres moyens et les hauteurs moyennes plus élevées des arbres révélés en savane soudanienne s'expliquent par la prédominance des individus âgés. Les faibles valeurs de diamètre moyen et de hauteur moyenne des arbres observées en savane guinéenne et subsoudanienne témoignent de la fréquence élevée d'individus jeunes. Ces observations pourraient être liées aux conditions climatiques et anthropiques (Rabiou et al., 2015b). En savane soudanienne, la réduction des régimes pluviométriques est à la base de la faible représentation des jeunes individus. Par contre, en savane guinéenne et subsoudanienne, les prélèvements excessifs ont pu contribuer à l'élimination des arbres de gros diamètres plus rentables pour l'exportation.

Cette étude montre que le vène est surexploité en Côte d'Ivoire. Il est donc nécessaire de renforcer la surveillance des aires protégées afin de réduire les pressions anthropiques qui menacent la survie de l'espèce. La protection dont bénéficie l'espèce en Côte d'Ivoire pourrait ne pas suffire à la préservation de celle-ci. Elle doit être renforcée par son inscription sur la liste rouge de l'Union internationale pour la conservation de la nature (UICN).

\section{Structure démographique des peuplements naturels de $P$. erinaceus}

Les structures en diamètre et en hauteur des peuplements naturels de $P$. erinaceus révèlent de manière générale une prédominance de jeunes individus $(1<c<3,6)$ en savane guinéenne et subsoudanienne. Les structures en diamètre, établies sur la base de la répartition des individus en classes de diamètre, sont caractérisées par un grand nombre de petits arbres et une réduction régulière du nombre d'individus d'une classe à la suivante. Par ailleurs, la structure démographique des arbres en classes de hauteur révèle une prédominance d'arbres de taille moyenne ( 6 à 16 m). Ces structures montrent une évolution positive des peuplements de $P$. erinaceus dans ces savanes où le renouvellement du peuplement est assuré (Feeley et al., 2007). Cela traduit une bonne capacité d'adaptation de l'espèce aux contraintes du milieu telles que les feux de brousse et les fluctuations des saisons des pluies de ces dernières décennies. De telles distributions ont été observées par Segla et al. (2015b) au Togo sur P. erinaceus et par Kakpo (2012) dans la forêt de Bonou au Bénin pour Afzelia africana. Ces structures témoignent aussi du prélèvement sélectif des arbres de plus gros diamètre pour les exportations pendant les exploitations forestières illégales. Par ailleurs, la faible fréquence d'arbres matures ou de semenciers (diamètre supérieur à $35 \mathrm{~cm}$ ) posera un problème de régénération sur le long terme dans ces peuplements (Yehouenou et al., 2012). 
En savane soudanienne, les individus de diamètre compris entre 15 et $45 \mathrm{~cm}$ sont prédominants avec de faibles fréquences d'individus de diamètre inférieur à $15 \mathrm{~cm}$. De plus, les arbres de hauteur comprise entre 10 et $16 \mathrm{~m}$ sont abondants. Ces observations montrent que $P$. erinaceus présente un déficit démographique de jeunes arbres dans cette zone (Abdourhamane et al., 2017). Ces structures témoignent d'un peuplement âgé en déclin naturel, avec un renouvellement insuffisant par les jeunes individus. Cela pourrait s'expliquer par les conditions climatiques défavorables de la zone. Les juvéniles rencontrent des difficultés à se maintenir pendant la longue saison sèche. De plus, les rares plantules qui émergent sont exposées aux herbivores qu'attirent les jeunes feuilles.

\section{Régénération naturelle de $P$. erinaceus}

Notre étude montre que $P$. erinaceus utilise plusieurs stratégies de régénération pour se multiplier. En effet, l'espèce se régénère par semis naturel, rejets de souche et drageons. La régénération par semis naturel et rejets de souche semble représenter les modes préférentiels de multiplication de l'espèce. Les drageons n'ont été observés qu'en savane guinéenne et subsoudanienne. Notre étude n'a pas révélé la présence de marcottes. Adjonou et al. (2010) ont toutefois montré que $P$. erinaceus pouvait également être multiplié par marcottes au Togo.

Ces résultats attestent que l'espèce est un bon candidat au reboisement des aires protégées dégradées. Cette faculté de l'espèce à se régénérer constitue une bonne opportunité pour la production de plants en pépinière. L'espèce se régénère aussi très bien après coupe (repousse). En effet, durant nos investigations, la quasi-totalité des coupes observées ont repoussé, quelle que fût la hauteur de coupe. Les rejets de souche assurent la pérennité des plantations au bénéfice de l'exploitant, comme dans les plantations de Tectona grandis, qui se reconstituent intégralement après la coupe. Des essais sylvicoles à base de jeunes plants de $P$. erinaceus sont donc envisageables pour restaurer les formations végétales naturelles dégradées ou pour le reboisement.

\section{Conclusion}

Cette étude a permis la caractérisation écologique des peuplements naturels de $P$. erinaceus dans les trois types de savane en Côte d'Ivoire. Les variations observées entre les différents peuplements sont dues en partie aux facteurs climatiques mais aussi à l'anthropisation (coupe, écorçage et émondage). L'analyse des paramètres dendrométriques a révélé que les savanes guinéenne et sub-soudanienne présentent les plus grandes valeurs de densité d'arbres et de densité de régénération. Ces savanes offrent des conditions environnementales plus favorables au développement des peuplements de $P$. erinaceus. Par contre, la savane soudanienne est caractérisée par des valeurs moyennes de diamètre, de hauteur totale et de hauteur de fût significativement plus élevées. La répartition des arbres de $P$. erinaceus en classes de diamètre et de hauteur a montré que la régénération naturelle de l'espèce est assurée en savane guinéenne et subsoudanienne avec une prédominance de jeunes individus. En savane soudanienne, les jeunes individus sont faiblement représentés. En ce qui concerne les stratégies de régénération, l'étude a montré que les semis naturels et les rejets de souche étaient plus abondants que les drageons.

Ces résultats indiquent que la protection effective des aires protégées pourrait favoriser la repousse des souches et, à long terme, la reconstitution des peuplements dégradés de $P$. erinaceus. Les semences peuvent être utilisées pour la production de plants en pépinière à grande échelle en sylviculture, le semis naturel étant naturellement le mode de régénération le plus répandu. Outre l'adoption de mesures de protection pour l'espèce, il semble urgent de promouvoir la mise en place de techniques de régénération assistée peu coûteuses en milieu naturel et de contrôler les pressions zoo-anthropiques qui menacent la survie de l'espèce.

\section{Remerciements}

Ces travaux ont été réalisés dans le cadre d'un doctorat de thèse unique. Les auteurs remercient la Société de développement des forêts (SODEFOR) pour les permis d'accès aux sites. Alice Goba remercie particulièrement le Programme d'appui stratégique à la recherche scientifique (PASRES) pour le financement de ses travaux de thèse et tous les gestionnaires et acteurs de la SODEFOR des sites prospectés.

\section{Références bibliographiques}

Abdourhamane H., Rabiou H., Diouf A., Morou B., Mahamane A., Bellefontaine R., 2017. Structure démographique et répartition spatiale des populations de Sclerocarya birrea (A. Rich.) Hochst. du secteur sahélien du Niger. Bois et Forêts des Tropiques, 333 (3) : 55-66. https://doi.org/10.19182/bft2017.333.a31468

Adjonou K., Ali N., Kokutse A. D., Novigno K. S., Kokou K., 2010. Étude de la dynamique des peuplements naturels de Pterocarpus erinaceus Poir. (Fabaceae) surexploités au Togo. Bois et Forêts des Tropiques, 306 (4) : 45-55. https://doi.org/10.19182/bft2010.306. a20431

Arbonnier M., 2004. Trees, shrubs and lianas of West African dry zones. Cirad, Margraf, MNHN, $574 \mathrm{p}$.

BNETD (Bureau national d'études techniques et de développement), 2015. Gestion durable des ressources forestières. Rapport pour les États généraux de la forêt, de la faune et des ressources en eau de Côte d'Ivoire, $89 \mathrm{p}$.

CITES (Convention sur le commerce international des espèces de faune et de flore menacées d'extinction), 2017. Examen des propositions d'amendement des annexes I et II. Dix-septième session de la Conférence des Parties à Johannesburg (Afrique du Sud), 24 septembre - 5 octobre 2016, 17 p.

CITES (Convention sur le commerce international des espèces de faune et de flore menacées d'extinction), 2015. Analyse du commerce international de Pterocarpus erinaceus et ses conséquences en Afrique de l'ouest. Document d'information. Vingt-deuxième réunion du comité des plantes à Tbilissi (Géorgie), 19-23 octobre 2015, $35 \mathrm{p}$.

FAO, 2002. Note thématique sur les Ressources Génétiques Forestières. Situation des ressources génétiques forestières de Côte d'Ivoire (zone de savanes). Rome, Italie, FAO, $47 \mathrm{p}$.

Feeley K. J., Davies J. S., Noor N. S., Rahman K. A., Tan S., 2007. Do current stem size distributions predict future populations changes? An empirical test of intraspecific patterns in tropical trees at two spatial scales. Journal of Tropical Ecology, 23: 191-198. 
Guillaumet J.-L., Adjanohoun E., 1971. La végétation de la Côte d'Ivoire. In : Avenard J.-M., Eldin M., Girard G., Sircoulon J., Touchebeuf P., Guillaumet J.-L., Adjanohoun E., Perraud A. (éds). Le milieu naturel de la Côte d'Ivoire. Paris, France, Orstom, Mémoires Orstom, $\mathrm{n}^{\circ}$ 50, 156-263. http://horizon.documentation.ird.fr/exl-doc/ pleins textes/pleins textes_6/Mem_cm/16368.pdf

Husch B., Beers T. W., Kershaw J. A. Jr., 2003. Forest Mensuration. Hoboken, NJ, USA, John Wiley, 4th edition, $443 \mathrm{p}$.

Interpol, 2016. Law enforcement and the West African Rosewood trade. "Comprendre et changer le commerce international : le cas de l'espèce ligneuse Pterocarpus erinaceus ». Bissau, Guinée-Bissau, 29-31 mars 2016.

Kakaï G. R L., Sinsin B., Palm R., 2008. Étude dendrométrique de Pterocarpus erinaceus Poir. des formations naturelles de la zone soudanienne au Bénin. Agronomie Africaine, 20 (3) : 245-255.

Kakpo S. B., 2012. Caractéristiques structurales et écologiques des forêts de Bonou et d'Itchede au sud-est du Bénin. Mémoire ingénieur agronome, Université d'Abomey-Calavi, Bénin, 70 p.

Koné M., Kouadio Y. L., Neuba D. F. R., Malan D. F., Coulibaly L., 2014. Évolution de la couverture forestière de la Côte d'Ivoire des années 1960 au début du 21e siècle. International Journal of Innovation and Applied Studies, 7 (2) : 782-794.

Mahamane A., Saadou M., 2008. Méthode d'étude de la flore et de la végétation tropicale. Projet SUN-EU. Actes de l'atelier de Niamey portant sur l'harmonisation des méthodes d'étude et d'analyse de la flore et de la végétation tropicale, $83 \mathrm{p}$.

MEDD (Ministère de l'Environnement et du Développement Durable), 2016. Plan d'investissement forestier de Côte d'Ivoire. Draft final. Abidjan, Côte d'Ivoire, Ministère de l'Environnement et du Développement durable, $85 \mathrm{p}$.

ONU, 2014. Rapport final du Groupe d'experts sur la Côte d'Ivoire établi en application du paragraphe 19 de la résolution 2101 (2013) du Conseil de sécurité (S/2014/266, 14th April 2014).

Ouattara D., Kouamé D., Tiebre M. S., Kouadio Y. J. C., N'guessan K. E., 2016. Biodiversité végétale et valeur d'usage en zone soudanienne de la Côte d'Ivoire. International Journal of Biological and Chemical Sciences, 10 (3) : 1122-1138.

Ouédraogo A., Adjima T., Hahn-Hadjali K., Guinko S., 2006. Diagnostic de l'état de dégradation des peuplements de quatre espèces ligneuses en zone soudanienne du Burkina Faso. Sécheresse, 17 (4) : 485-491.

Ouédraogo N., Sawadogo R. W., Tibiri A., Lompo M., Hay A. E., Koudou J., et al., 2011. Antioxidant, anti-inflammatory and analgesic activities of aqueous extract from stem bark of Pterocarpus erina ceus Poir. (Fabaceae). Journal of Medicinal Plants Research, 5 (10): 2047-2053.

Ouédraogo N., Sawadogo R. W., Tibiri A., Lompo M., Hay A. E., Koudou J., Dijoux M. G., Guissou I. P., 2012. Pharmacological properties and related constituents of stem bark of Pterocarpus erinaceus Poir. (Fabaceae). Asian Pacific Journal of Tropical Medicine, 5 (1): 46-51. Perraud A., 1971. Les sols. In : Avenard J.-M., Eldin M., Girard G., Sircoulon J., Touchebeuf P., Guillaumet J.-L., Adjanohoun E., Perraud A. (éds). Le milieu naturel de la Côte d'Ivoire. Paris, France, Orstom, Mémoires Orstom, n 50, 269-391. http://horizon.documentation. ird.fr/exl-doc/pleins textes/pleins textes 6/Mem_cm/16368.pdf Rabiou H., Diouf A., Bationo B. A., Mahamane A., Segla K. N., Adjonou K., et al., 2015a. Structure démographique de peuplement naturel et répartition spatiale des plantules de Pterocarpus erinaceus Poir. dans la forêt de Tiogo en zone soudanienne du Burkina Faso. International Journal of Biological and Chemical Sciences, 9 (1) : 69-81.

Rabiou H., Diouf A., Bationo B. A., Segla K. N., Adjonou K., Kokutse A. D., et al., 2015b. Structure des peuplements naturels de Pterocarpus erinaceus Poir. dans le domaine soudanien, au Niger et au Burkina Faso. Bois et Forêts des Tropiques, 325 (3) : 71-83. https:// doi.org/10.19182/bft2015.325.a31274

Rondeux J., 1999. La mesure des arbres et des peuplements forestiers. Gembloux, Belgique, Presses agronomiques de Gembloux, 522 p.

Roose E. J., 1979. Dynamique actuelle d'un sol ferrallitique gravillonnaire issu de granite sous culture et sous savane arbustive soudanienne du Nord de la Côte d'lvoire (Korhogo : 1967-1975). Cahiers Orstom, série Pédologie, 17 (2) : 81-118. https://core. ac.uk/download/pdf/39878754.pdf
Segla K. N., Adjonou K., Radji A. R., Kokutse A. D., Kokou K., Rabiou H., et al., 2015a. Importance socio-économique de Pterocarpus erinaceus Poir. au Togo. European Scientific Journal, 11 (23) : 1857-7881.

Segla K. N., Adjonou K., Rabiou H., Radji A. R., Kokutse A. D., Bationo B. A., et al., 2015b. Spatial distribution of Pterocarpus erinaceus Poir. (Fabaceae) natural stands in the sudanian and sudano-guinean zones of west Africa: gradient distribution and productivity variation across the five ecological zones of Togo. Annual Research \& Review in Biology, 6 (2): 89-102.

Silué N., Fofana J. I., Silue S., Diarrassouba N., Kouassi A. F., Kouakou K., 2014. Identification des espèces ligneuses utilisées dans l'alimentation des bovins dans la région du Poro (nord de la Côte d'Ivoire). Agronomie Africaine, 26 (3) : 217-229.

Sokpon N., Biaou S. H., Ouinsavi C., Hunhyet O., 2006. Bases techniques pour une gestion durable des forêts claires du Nord-Bénin : rotation, diamètre minimal d'exploitabilité et régénération. Bois et Forêts des Tropiques, 287 (1) : 45-57. https://doi.org/10.19182/ bft2006.287.a20322

Soro G. E., 2011. Modélisation statistique des pluies extrêmes en Côte d'Ivoire. Thèse unique, Université Nangui Abrogoua, Abidjan, Côte d'Ivoire, $173 \mathrm{p}$.

Sylla S. N., Samba R. T., Neyra M., Ndoye I., Giraud E., Willems A., De Lajudie P., Dreyfus B., 2002. Phenotypic and genotypic diversity of rhizobia nodulating $P$. erinaceus and $P$. lucens in Senegal. Systematic and Applied Microbiology, 25: 572-583.

Yao K. A. G., Koffi K. K., Ondo-Azi S. A., Baudoin J.-P., Zoro B. I. A., 2015. Seed yield component identification and analysis for exploiting recombinative heterosis in bottle gourd. International Journal of Vegetable Science, 21: 441-453.

Yehouenou T., Akouenou G., Ganglo J. C., 2012 Caractéristiques structurales et écologiques des populations d'Antiaris toxicaria (Pers.) Lesch et de Ceiba pentandra (L.) Gaertn dans les forêts reliques du Sud-Bénin. International Journal of Biological and Chemical Sciences, 6 (6) : 5056-5067.

\begin{tabular}{|c|c|}
\hline Rôle du contributeur & Noms des auteurs \\
\hline Conceptualisation & $\begin{array}{l}\text { R. S. Sié, G. K. Koffi, L. Kouonon, } \\
\text { A. Goba, Y. A. Koffi }\end{array}$ \\
\hline Gestion des données & A. Goba \\
\hline Analyse formelle & A. Goba \\
\hline $\begin{array}{l}\text { Acquisition } \\
\text { du financement }\end{array}$ & A. Goba, G. K. Koffi \\
\hline $\begin{array}{l}\text { Enquête } \\
\text { et investigation }\end{array}$ & A. Goba, A. Y. Koffi \\
\hline Méthodologie & A. Goba \\
\hline Gestion de projet & A. Goba, G. K. Koffi \\
\hline Ressources & A. Goba \\
\hline Supervision & R.S. Sié \\
\hline Validation & R. S. Sié, K. G. Koffi, L. Kouonon \\
\hline Visualisation & A. Goba \\
\hline $\begin{array}{l}\text { Écriture - Préparation } \\
\text { de l'ébauche originale }\end{array}$ & A. Goba \\
\hline $\begin{array}{l}\text { Écriture - Révision } \\
\text { et édition }\end{array}$ & $\begin{array}{l}\text { R. S. Sié, K. G. Koffi, L. Kouonon, } \\
\text { A. Goba }\end{array}$ \\
\hline
\end{tabular}

Bois et Forêts des Tropiques - Revue scientifique du Cirad
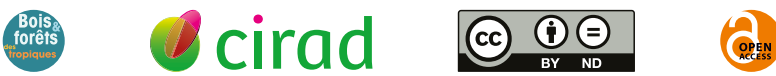

Cirad - Campus international de Baillarguet, 34398 Montpellier Cedex 5, France - Contact : bft@cirad.fr - ISSN : L-0006-579X 